\title{
Statistical Study of Monthly Rainfall Trends by Using the Transmuted Power Lindley Distribution
}

\author{
Daniele C. T. Granzotto ${ }^{1}$, Josmar Mazucheli ${ }^{1} \&$ Francisco Louzada $^{2}$ \\ ${ }^{1}$ Universidade Estadual de Maringá, DEs, PR, Brazil \\ ${ }^{2}$ Universidade de São Paulo, ICMC, SP, Brazil \\ Correspondence: Daniele C. T. Granzotto, Universidade Estadual de Maringá, DEs, PR, Brazil. E-mail: detgranzot- \\ to@uem.br
}

Received: February 3, 2016 Accepted: November 26, 2016 Online Published: December 21, 2016

doi:10.5539/ijsp.v6n1p111

URL: https://doi.org/10.5539/ijsp.v6n1p111

\begin{abstract}
In this article, we generalize the power Lindley distribution using a quadratic rank transmutation map to develop a transmuted power Lindley distribution. The new distribution exhibits, in addition to decreasing, increasing and bathtub hazard rate, depending on its parameters also unimodal hazard rate. A comprehensive mathematical properties of this distribution is provided. Some expressions for the moments, order statistics, quantiles function are derived. The model parameters are estimated by the maximum likelihood method. A Monte Carlo experiment on the finite sample behavior of the MLEs is performed. A real climatological data set was used in order to show the applicability of the new model and different statistics of fit were used as selection criteria.
\end{abstract}

Keywords: power Lindley distribution, transmutation map, reliability, rainfall data

\section{Introduction}

The Lindley distribution was proposed by Lindley (1958) and have been widely used in survival analysis and reliability fields. This distribution uses a mixture of exponential and length biased exponential distributions to illustrate the different between fiducial and posterior distributions. Late years, different applications and modifications have been proposed for this model such as: Ghitany et al. (2008) that argue that the Lindley distribution could be a better lifetime model than the exponential distribution through a numerical example; Nadarajah et al. (2011) and Zakerzadeh and Dolati (2009) in the proposition of a generalization; Merovci and Elbatal (2014) introduced a new lifetime distribution;Warahena-Liyanage and Pararai (2014) proposed an exponentiated power Lindley distribution with applications.

Those cited papers translate the concern with the proposition of new survival probability models based on the Lindley distribution. Also, various are the papers extending standard survival distributions in general, designed to serve as statistical survival models for a wide range of real lifetime phenomena. The challenge is the derivation of statistical survival probability models or simply survival distributions of real world lifetime phenomena that can represent more consistently the random behavior of experimental observations.

A convenient way to construct new distributions, in particular survival ones, are transmutation maps proposed by Shaw and Buckley (2007). The transmutation maps comprise the functional composition of a cumulative distribution function on a distribution with the inverse cumulative distribution (quantile) function of a non-Gaussian distributions, see for example Aryal and Tsokos $(2009,2011)$ that transmuted some models of Gamma distribution family and Granzotto and Louzada (2014); Louzada and Granzotto (2015) that proposed the transmuted log-logistic distribution and the regression extension of this model.

In this paper, we introduce a new lifetime distribution by transmuted and compounding power Lindley distribution named Transmuted Power Lindley (TPL) distribution. Briefly, it is the functional composition of a cumulative distribution function on a distribution with the inverse cumulative distribution (quantile) function of a non-Gaussian distribution, see Shaw and Buckley (2007). In this case, it incorporates a new third parameter (in our case ęË), what introduces a skewnwess and preserve the moments of the distribution base, see for example Shaw and Buckley (2007) and Granzotto and Louzada (2014). Although the TPL model is a positive distribution that can be applied for modeling on several areas such as reliability analysis, reliability along with engineer, hydrology, economics (income inequality) datasets; in this paper we proposed to analyse a real climatological dataset.

The paper is organized as follows. A background with the Lindley and its generalization are presented in Section 2 beyond the genesis of the transmutation map and the distributions Lindley and power Lindley. The derivation of the transmuted generalized Lindley distribution is presented in Section 3. Various important properties such as moments, 
moment generating function, quantiles, residual life, etc, for the transmuted Lindley distribution, as well as the minimum, maximum and median order statistics are presented in Section 4. Section 5 presents the maximum likelihood estimates and the asymptotic confidence intervals of the unknown parameters. In Section 6, the results of a simulation study is provided as well as the new distribution is illustrated in a climatological real data set, where we presented seven different statistics of fit were used as selection criteria. Final remarks are presented in Section 7.

\section{Background}

In this section we present a review of the Lindley and the Power Lindley distributions along with the transmutation map method, that are necessary to introduce the new model, TPL.

\subsection{The Lindley Distribution}

Proposed by Lindley (1958), the Lindley distribution is a exponential mixture that is important for studying stress-strength reliability modeling. Let $X$ be a nonnegative random variable denoting the lifetime of an individual in some population. The random variable $X$ is said to be Lindley distributed if the cumulative distribution function (c.d.f.) is given by

$$
F_{L}(x, \theta)=1-\left(1+\frac{\theta x}{\theta+1}\right) e^{-\theta x}, \quad x>0, \theta>0,
$$

and the corresponding probability density function (p.d.f.) is given by

$$
f_{L}(x, \theta)=\frac{\theta^{2}}{\theta+1}(1+x) e^{-\theta x} ; \quad x>0, \theta>0 .
$$

Ghitany et al. (2008) argue that the Lindley distribution could be a better lifetime model than the exponential distribution through a numerical example. In addition, they show that the hazard function of the Lindley distribution does not exhibit a constant hazard rate, indicating the flexibility of the Lindley distribution over the exponential distribution.

\subsection{The Power Lindley Distribution}

Ghitany et al. (2013) proposed new distribution, so called Power Lindley (GL) distribution, for modeling lifetime data. As the authors showed in their paper, they aim to discuss some properties of the power Lindley distribution which was formulated by using a power transformation $X=T^{1 / \alpha}$. The paper included the shapes of the density and hazard rate functions, the moments and some associated measures, the quantile function, and the limiting distributions of order statistics. Also, the maximum likelihood estimation of the model parameters and their asymptotic standard errors are derived.

Let $X$ be a nonnegative random variable denoting the lifetime of an individual in some population. The random variable $X$ is said to be power Lindley distributed with parameters $\theta$ and $\alpha$ if its cumulative density function (c.d.f.) is given by

$$
F_{P L}(x, \theta, \alpha)=\left[1-\left(1+\frac{\theta x^{\alpha}}{\theta+1}\right) e^{-\theta x^{\alpha}}\right],
$$

where $\theta>0$ and $\alpha>0$. The corresponding probability density function (p.d.f.) and the hazard (failure) rate function are given, respectively, by

$$
f_{P L}(x, \theta, \alpha)=\frac{\alpha \theta^{2}}{\theta+1}\left(1+x^{\alpha}\right) x^{\alpha-1} e^{-\theta x^{\alpha}} ; \quad x>0, \theta, \alpha>0
$$

and

$$
h_{P L}(x, \theta, \alpha)=\frac{\alpha \theta^{2}}{\theta+1+\theta x^{\alpha}}\left(1+x^{\alpha}\right) x^{\alpha-1} .
$$

Note that equation (3) has two parameters, $\theta$ and $\alpha$, just like the gamma, lognormal, Weibull and exponentiated exponential distributions. Note also that equation (5) has the attractive feature of allowing for monotonically decreasing, monotonically increasing and bathtub shaped hazard rate functions while not allowing for constant hazard rate functions.

\subsection{Transmutation Map}

Let $F_{1}$ and $F_{2}$ be the cumulative distribution functions, of two distributions with a common sample space. The general rank transmutation as given in ? is defined as

$$
G_{R 12}(u)=F_{2}\left(F_{1}^{-1}(u)\right) \text { and } G_{R 21}(u)=F_{1}\left(F_{2}^{-1}(u)\right) .
$$


Note that the inverse cumulative distribution function also known as quantile function is defined as

$$
F^{-1}(y)=\inf _{x \in R}\{F(x) \geq y\} \text { for } y \in[0,1] .
$$

The functions $G_{R 12}(u)$ and $G_{R 21}(u)$ both map the unit interval $I=[0,1]$ into itself, and under suitable assumptions are mutual inverses and they satisfy $G_{R i j}(0)=0$ and $G_{R i j}(1)=1$. A Quadratic Rank Transmutation Map (QRTM) is defined as

$$
G_{R 12}(u)=u+\lambda u(1-u),|\lambda| \leq 1,
$$

from which it follows that the cdf's satisfy the relationship

$$
F_{2}(x)=(1+\lambda) F_{1}(x)-\lambda F_{1}(x)^{2}
$$

which on differentiation yields,

$$
f_{2}(x)=f_{1}(x)\left[(1+\lambda)-2 \lambda F_{1}(x)\right],
$$

where $f_{1}(x)$ and $f_{2}(x)$ are the corresponding pdfs associated with cdf $F_{1}(x)$ and $F_{2}(x)$ respectively. An extensive information about the quadratic rank transmutation map is given in Shaw and Buckley (2007). Observe that at $\lambda=0$ we have the distribution of the base random variable. The following Lemma proved that the function $f_{2}(x)$ in given (8) satisfies the property of probability density function. Note that $f_{2}(x)$ given in (8) is a well defined probability density function. Rewriting $f_{2}(x)$ as $f_{2}(x)=f_{1}(x)\left[\left(1-\lambda\left(2 F_{1}(x)-1\right]\right.\right.$ we observe that $f_{2}(x)$ is nonnegative. We need to show that the integration over the support of the random variable is equal one. Consider the case when the support of $f_{1}(x)$ is $(-\infty, \infty)$. In this case we have

$$
\begin{aligned}
\int_{-\infty}^{\infty} f_{2}(x) d x & =\int_{-\infty}^{\infty} f_{1}(x)\left[(1+\lambda)-2 \lambda F_{1}(x)\right] d x \\
& =(1+\lambda) \int_{-\infty}^{\infty} f_{1}(x) d x-\lambda \int_{-\infty}^{\infty} 2 f_{1}(x) F_{1}(x) d x \\
& =(1+\lambda)-\lambda=1 .
\end{aligned}
$$

Similarly, other cases where the support of the random variable is a part of real line follows. Hence $f_{2}(x)$ is a well defined probability density function. We call $f_{2}(x)$ the transmuted probability density of a random variable with base density $f_{1}(x)$. Also note that when $\lambda=0$ then $f_{2}(x)=f_{1}(x)$.

\section{The Transmuted Power Lindley Distribution}

Let $X$ be a nonnegative random variable denoting the lifetime of an individual in some population. The random variable $X$ is said to be Transmuted Power Lindley (TPL) with parameters $\theta, \alpha$ and $\lambda$ if its cumulative density function (c.d.f.) is given by

$$
\begin{aligned}
F_{T P L}(x, \theta, \alpha, \lambda) & =G(x)[(1+\lambda)-\lambda G(x)] \\
& =\left[1-\left(1+\frac{\theta x^{\alpha}}{\theta+1}\right) e^{-\theta x^{\alpha}}\right]\left[1+\lambda\left(1+\frac{\theta x^{\alpha}}{\theta+1}\right) e^{-\theta x^{\alpha}}\right]
\end{aligned}
$$

where $\theta, \alpha>0$ and $\lambda \in(-1,1)$. The corresponding probability density function (p.d.f.) of the transmuted power Lindley is given by

$$
\begin{aligned}
f_{T P L}(x, \theta, \alpha, \lambda)= & \frac{\alpha \theta^{2}}{\theta+1}\left(1+x^{\alpha}\right) x^{\alpha-1} e^{-\theta x^{\alpha}} \\
& \times\left[1+\lambda-2 \lambda\left(1-\left(1+\frac{\theta x^{\alpha}}{\theta+1}\right) e^{-\theta x^{\alpha}}\right)\right] .
\end{aligned}
$$

The transmuted power Lindley distribution is an extended model to analyse data from complex situations and it generalizes some of the widely used distributions in reliability analysis. The power Lindley distribution is clearly a special case for $\lambda=0$ (see, Ghitany et al. (2013)). Also, the density and cumulative density curves of transmuted power model, for 
different parameters values can be seen in Figures 1, upper left and right panels, respectively.
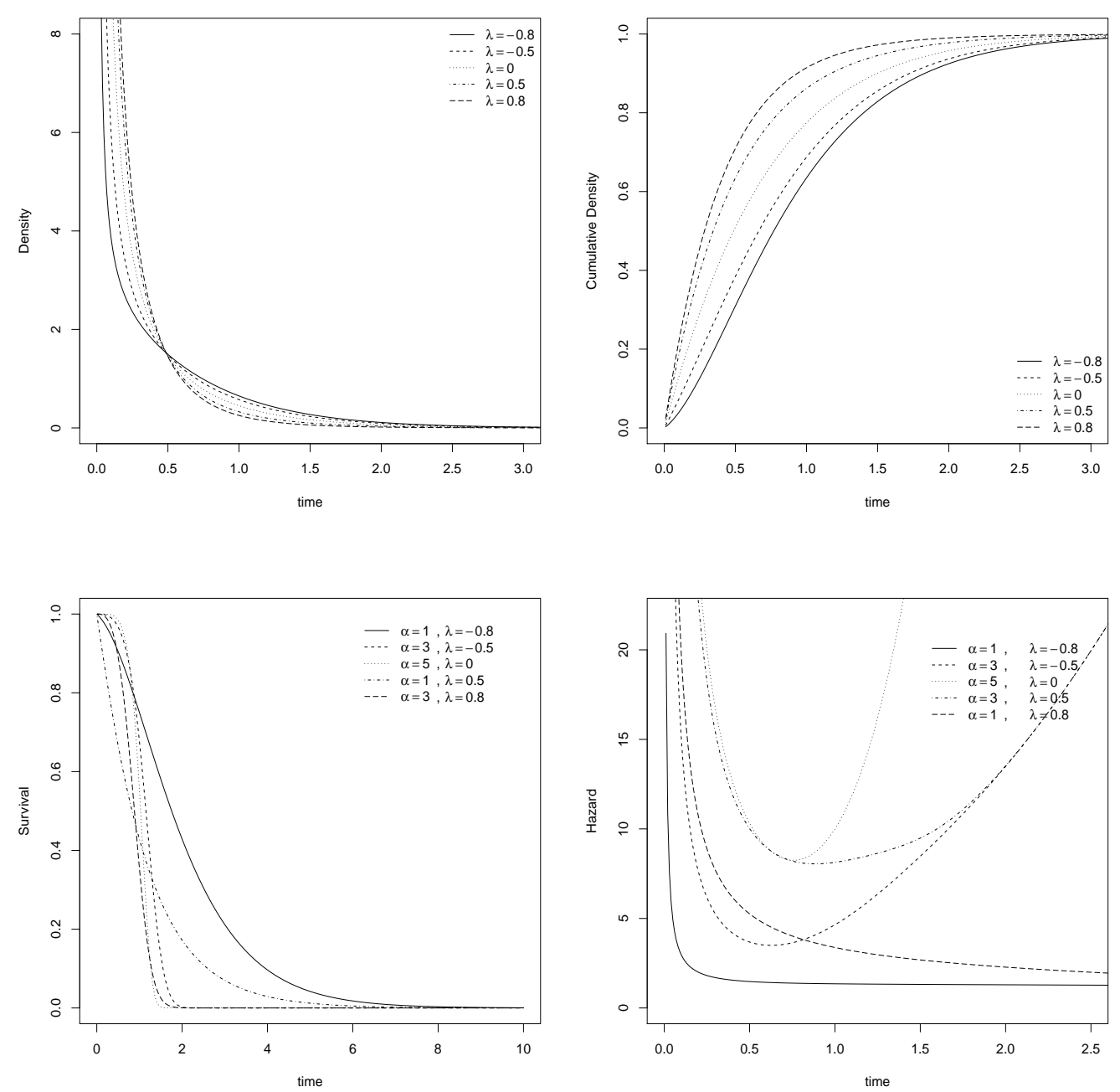

Figure 1. Upper panels: Density and Cumulative Density for fixed $\alpha=\theta=1$; Lower panels: Survival and Hazard curves for fixed $\theta=1$.

The reliability function of the transmuted power Lindley model is denoted by $R_{T P L}(t)$ and is defined as

$$
\begin{aligned}
R_{T P L}(t, \theta, \alpha, \lambda) & =1-F_{T P L}(t) \\
& =1-\left[1-\left(1+\frac{\theta x^{\alpha}}{\theta+1}\right) e^{-\theta x^{\alpha}}\right]\left[1+\lambda\left(1+\frac{\theta x^{\alpha}}{\theta+1}\right) e^{-\theta x^{\alpha}}\right] .
\end{aligned}
$$

For different parameters values the estimated curves can be seen in Figures 1, left lower panels. One of the characteristic in reliability analysis is the hazard rate function defined by

$$
h_{T P L}(t, \theta, \alpha, \lambda)=\frac{f_{T P L}(t)}{1-F_{T P L}(t)}
$$

It is important to note that the units for $h_{T P L}(t)$ is the probability of failure per unit of time, distance or cycles. These failure rates are defined with different choices of parameters, see Figures 1, right lower panels. 
The cumulative hazard function of the model is defined as

$$
\begin{aligned}
H_{T P L}(t, \theta, \alpha, \lambda)= & -\ln \mid 1-\left[1-\left(1+\frac{\theta x^{\alpha}}{\theta+1}\right) e^{-\theta x^{\alpha}}\right] \\
& \times\left[1+\lambda\left(1+\frac{\theta x^{\alpha}}{\theta+1}\right) e^{-\theta x^{\alpha}}\right] \mid
\end{aligned}
$$

It is important to note that the units for $H_{T P L}(t)$ is the cumulative probability of failure per unit of time, distance or cycles. For all choice of parameters the distribution has the decreasing patterns of cumulative instantaneous failure rates.

\section{Statistical Properties of TPL}

\subsection{Quantiles and Random Number Generation}

The quantile $x_{q}$ of the TPL is obtained from the following equation

$$
F\left(x_{q}\right)=\left[1-\left(1+\frac{\theta x_{q}^{\alpha}}{\theta+1}\right) e^{-\theta x_{q}^{\alpha}}\right]\left[1+\lambda\left(1+\frac{\theta x_{q}^{\alpha}}{\theta+1}\right) e^{-\theta x_{q}^{\alpha}}\right]=q
$$

setting $\phi=\left(1+\frac{\theta x_{q}^{\alpha}}{\theta+1}\right) e^{-\theta x_{q}^{\alpha}}$ then we have

$$
(1-\phi)(1+\lambda \phi)=q
$$

by solving the above equation with respect to $\phi$ we get

$$
\phi=\left[\frac{1-q}{\lambda}+\frac{(\lambda+1)^{2}}{4 \lambda^{2}}\right]^{1 / 2}+\frac{\lambda-1}{2 \lambda} .
$$

Hence we can obtain the quantile $x_{q}$ of the transmuted generalized Lindley as follows

$$
\left(1+\frac{\theta x_{q}^{\alpha}}{\theta+1}\right) e^{-\theta x_{q}^{\alpha}}=\left[\frac{1-q}{\lambda}+\frac{(\lambda+1)^{2}}{4 \lambda^{2}}\right]^{1 / 2}+\frac{\lambda-1}{2 \lambda} .
$$

The above equation has no closed form solution in $x_{q}$, so we have to use a numerical technique to get the quantiles. In particular, put $q=0.5$ in equation (15) one gets the median of transmuted power Lindley $(\alpha, \theta, \lambda, x)$.

Thus, random number generation as $x$ of the transmuted power Lindley $(\alpha, \theta, \lambda, x)$ is defined by the following relation

$$
\left[1-\left(1+\frac{\theta x_{q}^{\alpha}}{\theta+1}\right) e^{-\theta x_{q}^{\alpha}}\right]\left[1+\lambda\left(1+\frac{\theta x_{q}^{\alpha}}{\theta+1}\right) e^{-\theta x_{q}^{\alpha}}\right]=u
$$

where $u \sim U(0,1)$. This yields,

$$
\left(1+\frac{\theta x_{q}^{\alpha}}{\theta+1}\right) e^{-\theta x_{q}^{\alpha}}=\left[\frac{1-u}{\lambda}+\frac{(\lambda+1)^{2}}{4 \lambda^{2}}\right]^{1 / 2}+\frac{\lambda-1}{2 \lambda} .
$$

Equation (8) above does not have a closed form solution so we generate $u$ as uniform random variables from $\mathrm{U}(0,1)$ and solve for $x$ in order to generate random numbers from transmuted power Lindley distribution.

\subsection{Moments}

In this subsection we discuss the $r_{t h}$ moment for transmuted power Lindley distribution. Moments are necessary and important in any statistical analysis, especially in applications. It can be used to study the most important features and characteristics of a distribution (e.g., tendency, dispersion, skewness and kurtosis). 
Then, the $r_{t h}$ moment is given by

$$
\begin{aligned}
E\left(X^{r}\right)= & \int_{0}^{+\infty} x^{r} \frac{\alpha \theta^{2}}{\theta+1}\left(1+x^{\alpha}\right) x^{\alpha-1} e^{-\theta x^{\alpha}} \\
& \times\left[1+\lambda-2 \lambda\left(1-\left(1+\frac{\theta x^{\alpha}}{\theta+1}\right) e^{-\theta x^{\alpha}}\right)\right] d x \\
= & \frac{\alpha \theta^{2}}{\theta+1} \int_{0}^{+\infty}\left(1+x^{\alpha}\right) x^{r+\alpha-1} e^{-\theta x^{\alpha}}\left[1-\lambda+2 \lambda e^{-\theta x^{\alpha}}+\frac{2 \lambda \theta}{\theta+1} x^{\alpha} e^{-\theta x^{\alpha}}\right] .
\end{aligned}
$$

By using Gamma function, in order to solve the equation above, we have

$$
E\left(X^{r}\right)=\frac{\alpha \theta^{\frac{r-1}{\alpha}}}{\theta+1} \sum_{k=1}^{2} \theta^{1-k} \Gamma\left(\frac{r-1}{\alpha}+1+k\right)\left[1+\lambda 2^{-\left(\frac{r-1}{\alpha}+k\right)}\right] .
$$

The mean of the model and the variance are given, respectively by

$$
E(X)=\frac{\alpha}{\theta^{3}(\theta+1)}[\theta(2+\lambda)+(4+\lambda)]
$$

and

$$
\begin{aligned}
V(X)= & \frac{\alpha \theta^{\frac{1+2 \alpha}{\alpha}}}{\theta+1}\left\{\Gamma\left(\frac{1+2 \alpha}{\alpha}\right)\left(1+\lambda 2^{-(1+\alpha) / \alpha}\right)+\right. \\
& \left.\Gamma\left(\frac{1+3 \alpha}{\alpha}\right)\left(1+\lambda 2^{-(1+2 \alpha) / \alpha}\right)\right\}-\frac{\alpha^{2}}{\theta^{6}(\theta+1)^{2}}[\theta(2+\lambda)+(4+\lambda)]^{2}
\end{aligned}
$$

\subsection{Distribution of the Order Statistics}

According to Aryal and Tsokos (2011), suppose we have a system containing two components with each of them having independent and identical "base" distribution, for example power Lindley. If the components are connected in series then the overall system will have transmuted baseline distribution with $\lambda=1$ whereas if the components are parallel then the overall system will have a transmuted baseline.

It has been observed that a transmuted power Lindley distribution with $\lambda=1$ is the distribution of $\min \left(X_{1}, X_{2}\right)$ and a transmuted power Lindley distribution with $\lambda=-1$ is the distribution of the $\max \left(X_{1}, X_{2}\right)$ where $X_{1}$ and $X_{2}$ are independent and identically distributed 2-parameter power Lindley random variables.

In fact, the order statistics have many applications in reliability and life testing. The order statistics arise in the study of reliability of a system. Let $X_{1}, X_{2}, \ldots, X_{n}$ be a simple random sample from TPL $(\alpha, \theta, \lambda, x)$ with cumulative distribution function and probability density function as in (9) and (11), respectively. Let $X_{(1: n)} \leq X_{(2: n)} \leq \ldots \leq X_{(n: n)}$ denote the order statistics obtained from this sample. In reliability literature, $X_{(i: n)}$ denote the lifetime of an $(n-i+1)-$ out - of- $n$ system which consists of $n$ independent and identically components. Then the pdf of $X_{(i: n)}, 1 \leq i \leq n$ is given by

$$
f_{i: n}(x)=\frac{1}{\beta(i, n-i+1)}\left[F\left(x_{(i)}\right)\right]^{i-1}\left[1-F\left(x_{(i)}\right)\right]^{n-i} f\left(x_{(i)}\right)
$$

also, the joint pdf of $X_{(i: n)}, X_{(j: n)}$ and $1 \leq i \leq j \leq n$ is

$$
f_{i:: j: n}\left(x_{i}, x_{j}\right)=C\left[F\left(x_{i}\right)\right]^{i-1}\left[F\left(x_{j}\right)-F\left(x_{i}\right)\right]^{j-i-1}\left[1-F\left(x_{j}\right)\right]^{n-j} f\left(x_{i}\right) f\left(x_{j}\right)
$$

where

$$
C=\frac{n !}{(i-1) !(j-i-1) !(n-j) !} .
$$

We defined the first order statistics $X_{(1)}=\min \left(X_{1}, X_{2}, \ldots, X_{n}\right)$, the the last order statistics as $X_{(n)}=\max \left(X_{1}, X_{2}, \ldots, X_{n}\right)$ and median order $X_{m+1}$. 


\subsection{Distribution of Minimum, Maximum and Median}

Let $X_{(1: n)} \leq X_{(2: n)} \leq \ldots \leq X_{(n: n)}$ be independently identically distributed order random variables from the transmuted generalized Lindley distribution having first, last and median order probability density function are given by the following

$$
\begin{aligned}
f_{1: n}(x)= & n\left[1-F\left(x_{(1)}\right)\right]^{n-1} f\left(x_{(1)}\right) \\
= & n\left[\frac{n \alpha \theta^{2}}{\theta+1}\left(1+x_{(1)}^{\alpha}\right) x_{(1)}^{\alpha-1} e^{-\theta x_{(1)}^{\alpha}}\right. \\
& \left.\times\left[1+\lambda-2 \lambda\left(1-\zeta_{(1)}\right)\right]\left[\left(1-\left(1-\zeta_{(1)}\right)\right)\left(1-\lambda \zeta_{(1)}\right)\right]^{n-1}\right],
\end{aligned}
$$

where

$$
\begin{aligned}
\zeta_{(i)}=\left(1+\frac{\theta x_{(i)}^{\alpha}}{\theta+1}\right) e^{-\theta x_{(i)}^{\alpha}} \\
\left.f_{n: n}(x)=n\left[F\left(x_{(n)}, \Phi\right)\right]^{n-1} f\left(x_{(n)}\right), \Phi\right) \\
=n\left[\frac{n \alpha \theta^{2}}{\theta+1}\left(1+x_{(n)}^{\alpha}\right) x_{(n)}^{\alpha-1} e^{-\theta x_{(n)}^{\alpha}}\right. \\
\left.\times\left[1+\lambda-2 \lambda\left(1-\zeta_{(n)}\right)\right]\left[\left(1-\left(1-\zeta_{(n)}\right)\right)\left(1-\lambda \zeta_{(n)}\right)\right]^{n-1}\right]
\end{aligned}
$$

and

$$
\begin{aligned}
f_{m+1: n}(\widetilde{x})= & \frac{(2 m+1) !}{m ! m !}(F(\widetilde{x}))^{m}(1-F(\widetilde{x}))^{m} f(\widetilde{x}) \\
= & \frac{(2 m+1) !}{m ! m !}\left[\left(1-\zeta_{(m+1)}\right)\left(1+\lambda \zeta_{(m+1)}\right)\right]^{m} \\
& \times\left[\frac{\alpha \theta^{2}}{\theta+1}\left(1+x_{(m+1)}^{\alpha}\right) x_{(m+1)}^{\alpha-1} e^{-\theta x_{(m+1)}^{\alpha}}\right. \\
& \left.\times\left[1+\lambda-2 \lambda\left(1-\zeta_{(m+1)}\right)\right]\left[\left(1-\left(1-\zeta_{(m+1)}\right)\right)\left(1-\lambda \zeta_{(m+1)}\right)\right]^{m}\right] .
\end{aligned}
$$

We notice that the minimum, maximum and median order statistics of three parameters transmuted power Lindley distribution have different life time distributions when its parameters are changed.

\section{Inference}

In this section we consider the maximum likelihood estimators (MLE's) of transmuted power Lindley distribution. Let $\phi=(\alpha, \theta, \lambda)^{T}$,in order to estimate the parameters $\alpha, \theta$, and $\lambda$ of transmuted power Lindley distribution, let $x_{1}, \ldots, x_{n}$ be a random sample of size $n$ from $\operatorname{TPL}(\alpha, \theta, \lambda)$, we obtain the likelihood function as follows

$$
\begin{aligned}
L(\alpha, \theta, \lambda)= & \left(\frac{\alpha \theta^{2}}{\theta+1}\right)^{n} \prod_{i=1}^{n}\left(1+x_{i}^{\alpha}\right) x_{i}^{\alpha-1} e^{-\theta x_{i}^{\alpha}} \\
& \times\left[1+\lambda-2 \lambda\left(1-\left(1+\frac{\theta x_{i}^{\alpha}}{\theta+1}\right) e^{-\theta x_{i}^{\alpha}}\right)\right],
\end{aligned}
$$

then the log likelihood function can be written as

$$
\begin{aligned}
\ln L(\alpha, \theta, \lambda)= & n \ln \alpha+2 n \ln \theta-n \ln (1+\theta)+\sum_{i=1}^{n} \ln \left(1+x_{i}^{\alpha}\right) \\
& +(\alpha-1) \sum_{i=1}^{n} \ln x_{i}-\theta \sum_{i=1}^{n} x_{i}^{\alpha}+ \\
& +\sum_{i=1}^{n} \ln \left[1+\lambda-2 \lambda\left(1-\omega_{i} e^{-\theta x_{i}^{\alpha}}\right)\right]
\end{aligned}
$$


where

$$
\omega_{i}=\left(1+\frac{\theta x_{i}^{\alpha}}{\theta+1}\right) .
$$

Differentiating $\ln L(\alpha, \theta, \lambda)$ with respect to each parameter $\alpha, \theta$, and $\lambda$ and setting the result equals to zero, we obtain maximum likelihood estimates. The partial derivatives of $\ln L(\alpha, \theta, \lambda)$ with respect to each parameter or the score function is given by

$$
U_{n}(\phi)=\left(U_{\alpha}, U_{\theta}, U_{\lambda}\right)^{T}
$$

where

$$
\begin{aligned}
U_{\alpha}= & \frac{\partial \ln L}{\partial \alpha}=\frac{n}{\alpha}+\sum_{i=1}^{n} \frac{x_{i}^{\alpha} \ln \left(x_{i}\right)}{\left(1+x_{i}^{\alpha}\right)}+(1-\theta) \sum_{i=1}^{n} \ln x_{i} \\
& -2 \lambda \sum_{i=1}^{n}\left\{\frac{\theta x_{i}^{\alpha} \ln \left(x_{i}\right) e^{-\theta x_{i}^{\alpha}}\left[\omega_{i}-\frac{1}{1+\theta}\right]}{1+\lambda-2 \lambda\left(1-\omega_{i} e^{-\theta x_{i}^{\alpha}}\right)}\right\}, \\
U_{\theta}= & \frac{\partial \ln L}{\partial \theta}=\frac{n(2+\theta)}{\theta(1+\theta)}-\sum_{i=1}^{n} \ln x_{i}^{\alpha} \\
& -2 \lambda \sum_{i=1}^{n} x_{i}^{\alpha} e^{-\theta x_{i}^{\alpha}}\left\{\frac{\omega_{i}-(1+\theta)^{-2}}{1+\lambda-2 \lambda\left(1-\omega_{i} e^{-\theta x_{i}^{\alpha}}\right)}\right\}
\end{aligned}
$$

and

$$
U_{\lambda}=\frac{\partial \ln L}{\partial \lambda}=\sum_{i=1}^{n} \frac{2 \omega_{i} e^{-\theta x_{i}^{\alpha}}-1}{1+\lambda-2 \lambda\left(1-\omega_{i} e^{-\theta x_{i}^{\alpha}}\right)} .
$$

The maximum likelihood estimation $\widehat{\phi}=(\widehat{\alpha}, \widehat{\theta}, \widehat{\lambda})^{T}$ of $\phi=(\alpha, \theta, \lambda)^{T}$ is obtained by solving the non linear equations $U_{n}(\phi)=0$. These equations cannot be solved analytically but statistical software can be used to solve them numerically. For interval estimation and hypothesis tests on the model parameters, we require the information matrix. The $3 \times 3$ observed information matrix is given by

$$
I_{n}(\varphi)=-\left[\begin{array}{ccc}
I_{\alpha \alpha} & I_{\alpha \theta} & I_{\alpha \lambda} \\
I_{\theta \alpha} & I_{\theta \theta} & I_{\theta \lambda} \\
I_{\lambda \alpha} & I_{\lambda \theta} & I_{\lambda \lambda}
\end{array}\right]
$$

where $I_{n}(\phi)=\frac{\partial^{2} \ln L}{\partial \phi \partial \phi^{T}}$. Applying the usual large sample approximation, MLE of $\phi$, i.e $\widehat{\phi}$ can be treated as being approximately $N_{3}\left(\phi, J_{n}(\phi)^{-1}\right)$, where $J_{n}(\phi)=E\left[I_{n}(\phi)\right]$. Under conditions that are fulfilled for parameters in the interior of the parameter space but not on the boundary, the asymptotic distribution of $\sqrt{n}(\widehat{\phi}-\phi)$ is $N_{3}\left(0, J(\varphi)^{-1}\right)$, where $J(\phi)=$ $\lim _{n \rightarrow \infty} n^{-1} I_{n}(\phi)$ is the unit information matrix. This asymptotic behavior remains valid if $J(\phi)$ is replaced by the average sample information matrix evaluated at $\widehat{\phi}$, say $n^{-1} I_{n}(\widehat{\phi})$. The estimated asymptotic multivariate normal $N_{3}\left(\phi, I_{n}(\widehat{\phi})^{-1}\right)$ distribution of $\widehat{\phi}$ can be used to construct approximate confidence intervals for the parameters and for the hazard rate and survival functions. An 100\% $(1-\gamma)$ asymptotic confidence interval for each parameter $\phi_{r}$ is given by

$$
A C I_{r}=\left(\widehat{\phi}_{r}-z_{\frac{\gamma}{2}} \sqrt{\widehat{I}_{r r}}, \widehat{\phi}_{r}+z_{\frac{\gamma}{2}} \sqrt{\widehat{I_{r r}}}\right),
$$

where $\widehat{I_{r r}}$ is the $(r, r)$ diagonal element of $I_{n}(\widehat{\varphi})^{-1}$ for $r=1,2,3$, and $z_{\frac{\gamma}{2}}$ is the quantile $1-\frac{\gamma}{2}$ of the standard normal distribution.

In order to compare the models seven different statistics of fit were used as selection criteria in Section 6.2 : $-2 \times$ log-likelihood (Neg2LogLike), Akaike's information criterion (AIC), corrected Akaike's information criterion (AICC), Kolmogorov-Smirnov statistic (KS), Anderson-Darling statistic (AD) and Cramér-von-Mises statistic (CvM).

The first ones, AIC and AICC, are widely used in reliability analysis as a selection criteria. The AIC can be obtained by using the following expression:

$$
A I C=-2 \ln L_{M}(\zeta)+2 p
$$


with $L_{M}$ the likelihood of the model $M, \zeta$ the vector of parameters to the model $M$ and $p$ the number of parameters to the model $M$. The AICC is given, respectively, by

$$
A I C C=-2 \ln L_{M}(\zeta)+\frac{2 p(p+1)}{n-p-1}
$$

with $n$ the number of observations.

Further, the Anderson-Darling and the Cramér-von Mises statistics are widely utilized to determine how closely a specific distribution whose associated cumulative distribution function fits the empirical distribution associated with a given data set. These statistics are

$$
A^{*}=\left(\frac{9}{4 n^{2}}+\frac{3}{4 n}+1\right)\left[n+\frac{1}{n} \sum_{j=1}^{n}(2 j-1) \log \left[z_{i}\left(1-z_{n-j+1}\right)\right]\right]
$$

and

$$
W^{*}=\left(\frac{1}{2 n}+1\right)\left[\sum_{j=1}^{n}\left(z_{i}-\frac{2 j-1}{2 n}\right)^{2}+\frac{1}{12 n}\right],
$$

respectively, $z_{i}=F\left(y_{j}\right)$, where the $y_{j}$ values being the ordered observations. The smaller these statistics are, the better the fit. Upper tail percentiles of the asymptotic distributions of these goodness-of-fit statistics were tabulated in Nichols and Padgett (2006).

\section{Data Experiments}

This section presents the results of a Monte Carlo experiment on the finite sample behavior of the MLEs as well as illustrate the applicability of the proposed distribution in various real data sets on rainfall.

\subsection{Simulation Study}

The Monte Carlo simulation results were obtained from 1,000 Monte Carlo replications. The sample sizes $n$ range from 30 to 500, generated according to a transmuted power Lindley distribution for each combination of the parameter values $\alpha, \lambda$ and $\theta=2$ fixed. Table 1 shows that the estimates and BIAS of the MLEs and Table 2 shows us the coverage probabilities of a $95 \%$ two sided confidence intervals for the model parameters and the mean square error which decrease with the increasing of the sample size. 
Table 1. Parameters estimated and BIAS of the MLEs.

\begin{tabular}{c|cc|ccc|ccc}
\hline Sample & \multicolumn{2}{|c|}{ Generated } & \multicolumn{3}{|c|}{ Estimate } & \multicolumn{3}{c}{ BIAS } \\
\cline { 2 - 9 } Size & $\alpha$ & $\lambda$ & $\theta$ & $\alpha$ & $\lambda$ & $\theta$ & $\alpha$ & $\lambda$ \\
\hline 30 & 3.0 & -0.5 & 2.191 & 2.877 & -0.278 & 0.191 & 0.123 & 0.222 \\
50 & 3.0 & -0.5 & 2.173 & 2.783 & -0.292 & 0.173 & 0.217 & 0.208 \\
100 & 3.0 & -0.5 & 2.126 & 2.753 & -0.258 & 0.126 & 0.247 & 0.242 \\
300 & 3.0 & -0.5 & 2.119 & 2.747 & -0.255 & 0.119 & 0.253 & 0.245 \\
500 & 3.0 & -0.5 & 2.103 & 2.757 & -0.237 & 0.103 & 0.243 & 0.263 \\
\hline 30 & 8.0 & -0.5 & 2.010 & 8.396 & -0.421 & 0.010 & 0.396 & 0.079 \\
50 & 8.0 & -0.5 & 2.004 & 8.245 & -0.442 & 0.004 & 0.245 & 0.058 \\
100 & 8.0 & -0.5 & 1.988 & 8.142 & -0.445 & 0.012 & 0.142 & 0.055 \\
300 & 8.0 & -0.5 & 1.986 & 8.066 & -0.458 & 0.014 & 0.066 & 0.042 \\
500 & 8.0 & -0.5 & 1.977 & 8.089 & -0.448 & 0.023 & 0.088 & 0.052 \\
\hline 30 & 5.0 & 0.5 & 2.12267 & 5.0985 & 0.47831 & 0.12267 & 0.09846 & 0.02169 \\
50 & 5.0 & 0.5 & 2.11252 & 5.0225 & 0.45167 & 0.11252 & 0.0225 & 0.04833 \\
100 & 5.0 & 0.5 & 2.08432 & 4.9765 & 0.44898 & 0.08432 & 0.02345 & 0.05102 \\
300 & 5.0 & 0.5 & 2.05484 & 4.9635 & 0.46933 & 0.05484 & 0.03649 & 0.03067 \\
500 & 5.0 & 0.5 & 2.05772 & 4.9686 & 0.46895 & 0.05772 & 0.03143 & 0.03105 \\
\hline 30 & 3.0 & -0.8 & 1.93496 & 3.2891 & -0.75086 & 0.06504 & 0.28911 & 0.04914 \\
50 & 3.0 & -0.8 & 1.92625 & 3.2141 & -0.76083 & 0.07375 & 0.21406 & 0.03917 \\
100 & 3.0 & -0.8 & 1.93109 & 3.1594 & -0.77346 & 0.06891 & 0.15942 & 0.02654 \\
300 & 3.0 & -0.8 & 1.94909 & 3.0946 & -0.81174 & 0.05091 & 0.0946 & 0.01174 \\
500 & 3.0 & -0.8 & 1.96397 & 3.0639 & -0.83572 & 0.03603 & 0.06387 & 0.03572 \\
\hline 30 & 8.0 & 0.2 & 2.20698 & 8.0716 & 0.40377 & 0.20698 & 0.07161 & 0.09623 \\
50 & 8.0 & 0.2 & 2.21645 & 7.9576 & 0.36802 & 0.21645 & 0.04238 & 0.13198 \\
100 & 8.0 & 0.2 & 2.24932 & 7.9094 & 0.3096 & 0.24932 & 0.09061 & 0.1904 \\
300 & 8.0 & 0.2 & 2.30488 & 7.8905 & 0.25473 & 0.30488 & 0.10948 & 0.24527 \\
500 & 8.0 & 0.2 & 2.31874 & 7.8921 & 0.2423 & 0.31874 & 0.10792 & 0.2577 \\
\hline & & & & & & & &
\end{tabular}


Table 2. Mean square error (mse) of the MLEs and Coverage Probability.

\begin{tabular}{c|cc|ccc|ccc}
\hline Sample & \multicolumn{3}{|c|}{ Generated } & \multicolumn{4}{|c|}{ MSE } & \multicolumn{3}{|c}{ Coverage Probability } \\
\cline { 2 - 9 } Size & $\alpha$ & $\lambda$ & $\theta$ & $\alpha$ & $\lambda$ & $\theta$ & $\alpha$ & $\lambda$ \\
\hline 30 & 3.0 & -0.5 & 0.715 & 0.921 & 0.834 & 0.998 & 0.881 & 0.859 \\
50 & 3.0 & -0.5 & 0.579 & 0.713 & 0.719 & 0.988 & 0.860 & 0.864 \\
100 & 3.0 & -0.5 & 0.439 & 0.524 & 0.591 & 0.972 & 0.900 & 0.920 \\
300 & 3.0 & -0.5 & 0.263 & 0.328 & 0.367 & 0.986 & 0.904 & 0.966 \\
500 & 3.0 & -0.5 & 0.201 & 0.251 & 0.301 & 0.987 & 0.920 & 0.992 \\
\hline 30 & 8.0 & -0.5 & 0.637 & 3.916 & 0.863 & 0.977 & 0.971 & 0.749 \\
50 & 8.0 & -0.5 & 0.527 & 3.186 & 0.722 & 0.985 & 0.939 & 0.797 \\
100 & 8.0 & -0.5 & 0.410 & 2.478 & 0.560 & 0.988 & 0.910 & 0.857 \\
300 & 8.0 & -0.5 & 0.272 & 1.501 & 0.374 & 0.982 & 0.919 & 0.891 \\
500 & 8.0 & -0.5 & 0.228 & 1.199 & 0.317 & 0.974 & 0.930 & 0.916 \\
\hline 30 & 5.0 & 0.5 & 0.84692 & 1.4552 & 0.79661 & 0.942 & 0.978 & 0.752 \\
50 & 5.0 & 0.5 & 0.74045 & 1.036 & 0.69788 & 0.913 & 0.969 & 0.82 \\
100 & 5.0 & 0.5 & 0.60588 & 0.7043 & 0.58288 & 0.886 & 0.973 & 0.86 \\
300 & 5.0 & 0.5 & 0.43729 & 0.3893 & 0.41779 & 0.89 & 0.95 & 0.886 \\
500 & 5.0 & 0.5 & 0.3756 & 0.3079 & 0.35627 & 0.916 & 0.949 & 0.908 \\
\hline 30 & 3.0 & -0.8 & 0.45726 & 1.0588 & 0.82622 & 0.966 & 0.96 & 0.453 \\
50 & 3.0 & -0.8 & 0.35881 & 0.7789 & 0.63423 & 0.947 & 0.961 & 0.515 \\
100 & 3.0 & -0.8 & 0.27257 & 0.5424 & 0.42326 & 0.955 & 0.938 & 0.676 \\
300 & 3.0 & -0.8 & 0.15113 & 0.2901 & 0.18577 & 0.953 & 0.956 & 0.811 \\
500 & 3.0 & -0.8 & 0.10251 & 0.1957 & 0.11576 & 0.97 & 0.983 & 0.834 \\
\hline 30 & 8.0 & 0.2 & 0.89199 & 2.8335 & 0.8573 & 0.924 & 0.981 & 0.723 \\
50 & 8.0 & 0.2 & 0.78179 & 1.9179 & 0.76533 & 0.928 & 0.971 & 0.799 \\
100 & 8.0 & 0.2 & 0.67178 & 1.3264 & 0.65662 & 0.920 & 0.961 & 0.859 \\
300 & 8.0 & 0.2 & 0.52796 & 0.7569 & 0.48225 & 0.920 & 0.973 & 0.908 \\
500 & 8.0 & 0.2 & 0.4532 & 0.5759 & 0.40603 & 0.956 & 0.97 & 0.924 \\
\hline
\end{tabular}

\subsection{Applications}

In this section we fit, by using the maximum likelihood method, the transmuted power Lindley distribution (TPL) to rainfall data from six weather stations located in Santa Catarina state, Brazil. The data consist of monthly rainfall for the years from 1971 to 2014. and were obtained from the National Institute of Meteorology at website

http://www.inmet.gov.br/portal/index.php?r=bdmep/bdmep. Table 3 gives the latitude, longitude, observed period and the number of valid observations in that period.

Also, for comparison proposes, we have considered four alternative distributions: the one parameter Lindley distribution (L) with $f(y \mid \theta)=\frac{\theta^{2}}{1+\theta}(1+y) e^{-\theta y}$, the weighted Lindley distribution (WL) with $f(y \mid \theta, \lambda)=\frac{\theta^{l+1}}{(\theta+\lambda) \Gamma(\lambda)} y^{\lambda-1}(1+y) e^{-\theta y}$, the power Lindley distribution (PL) with $f(y \mid \theta, \lambda)=\frac{\lambda \theta^{2}}{1+\theta}\left(1+y^{\lambda}\right) y^{\lambda-1} e^{-\theta y^{\lambda}}$ and the transmuted Lindley distribution (TL) with $f(y \mid \theta, \lambda)=\frac{\theta^{2}}{1+\theta}(1+y) e^{-\theta y}\left[1-\lambda+2 \lambda\left(1+\frac{\theta y}{1+\theta}\right) e^{-\theta y}\right]$. As a complement, Figure 3 presents a PPlot of the adjusted models.

In Table 5 we presented, for all models and data sets, the maximum likelihood and standard errors estimates for $\theta, \alpha$ and $\lambda$. The maximum likelihood estimates were obtained by SAS/SEVERITY procedure, SAS (2011). The SAS/SEVERITY procedure can fit multiple distributions at the same time and choose the best distribution according to a specified selection criterion. Seven different statistics of fit were used as selection criteria: $-2 \times \log$-likelihood (Neg2LogLike), AIC, AICC, $\mathrm{KS}, \mathrm{AD}$ and CvM. The calculated values of theses statistics are reported in Table 4 which present the superscripts that indicates the rank obtained by the distribution according to the selection criteria (the smaller the better). The column labeled as "RT" shows the sum of the ranks. From the values of "RT" column we can see that the TPL distribution is judged as being the most appropriate for five data sets. The fitted transmuted power Lindley density is displayed in Figure 2. 

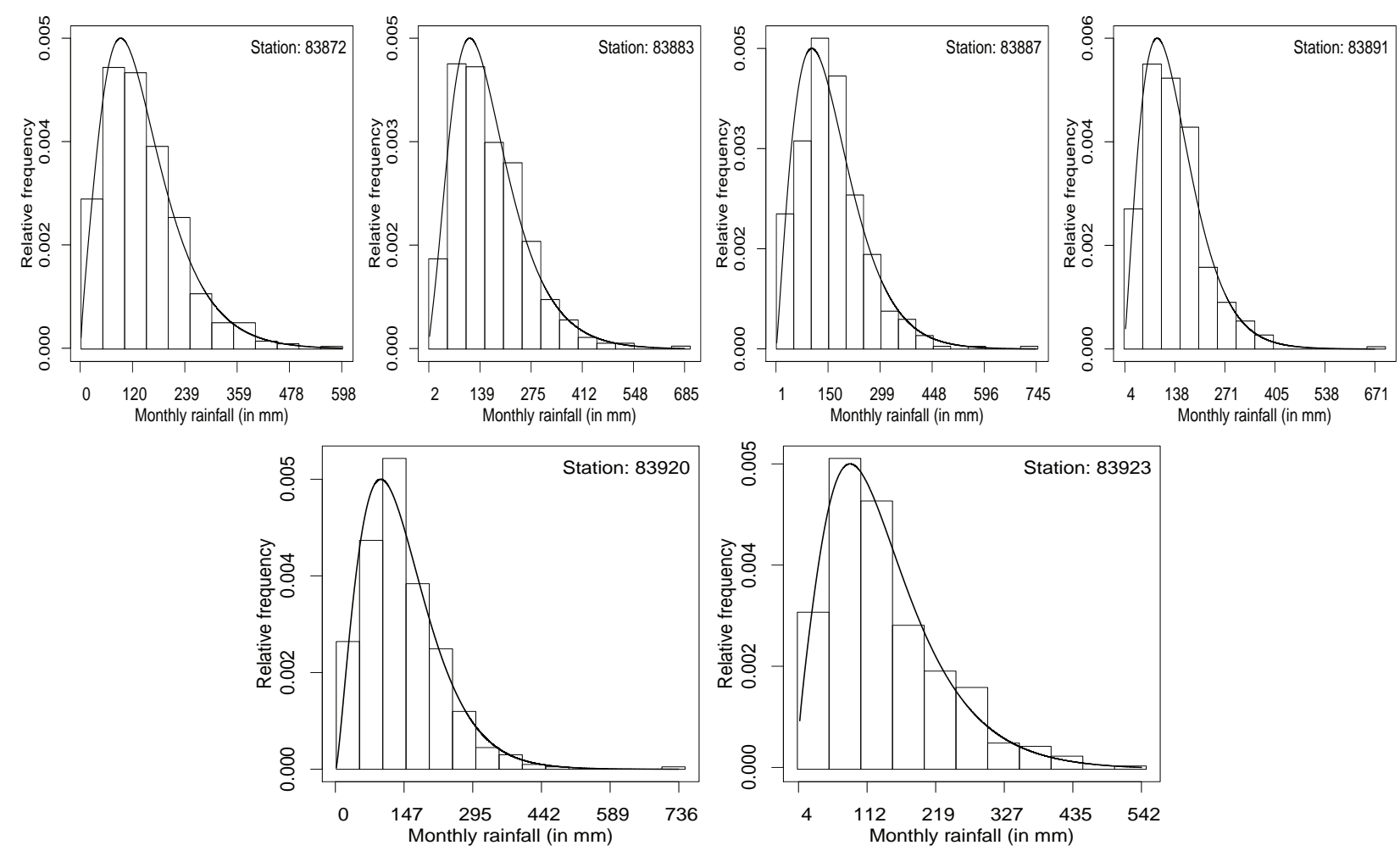

Figure 2. Adjusted TPL model.

Table 3. Description of meteorological stations.

\begin{tabular}{cccccc}
\hline Location & Latitude & Longitude & begin & end & $\mathrm{n}$ \\
\hline Indaial (OMM: 83872) & -26.90 & -49.21 & $31 / 12 / 1970$ & $31 / 12 / 2014$ & 443 \\
Chapeco (OMM: 83883) & -27.11 & -52.61 & $31 / 07 / 1973$ & $31 / 12 / 2014$ & 434 \\
Campos Novos (OMM: 83887) & -27.38 & -51.20 & $31 / 01 / 1970$ & $31 / 12 / 2014$ & 468 \\
Lages (OMM: 83891) & -27.81 & -50.33 & $31 / 01 / 1970$ & $31 / 12 / 2014$ & 467 \\
São Joaquim (OMM: 83920) & -28.30 & -49.93 & $31 / 01 / 1970$ & $31 / 12 / 2014$ & 447 \\
Urussanga (OMM: 83923) & -28.51 & -49.31 & $31 / 01 / 1970$ & $31 / 07 / 2014$ & 344 \\
\hline
\end{tabular}


Table 4. Model selection.

\begin{tabular}{|c|c|c|c|c|c|c|c|c|}
\hline Station & Model & Jeg2LogLike & AIC & AICC & KS & $\mathrm{AD}$ & $\mathrm{CvM}$ & RT \\
\hline \multirow{5}{*}{83872} & $\mathrm{~L}$ & 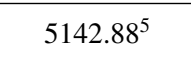 & $5144.88^{5}$ & $5144.89^{5}$ & $1.36^{5}$ & $2.92^{5}$ & $0.50^{5}$ & $34^{5}$ \\
\hline & WL & 5137.8 & $5141.86^{4}$ & $5141.89^{4}$ & $0.88^{3}$ & $1.03^{3}$ & $0.16^{3}$ & $26^{4}$ \\
\hline & PL & $5134.41^{3}$ & $5138.41^{3}$ & $5138.44^{3}$ & $0.73^{2}$ & $0.61^{2}$ & $0.08^{2}$ & $17^{2}$ \\
\hline & $\mathrm{TL}$ & $5134.26^{2}$ & $5138.26^{2}$ & $5138.29^{2}$ & $0.95^{4}$ & $1.25^{4}$ & $0.20^{4}$ & $19^{3}$ \\
\hline & TPL & $5128.66^{1}$ & $5134.66^{1}$ & $5134.71^{1}$ & $0.67^{1}$ & $0.35^{1}$ & $0.05^{1}$ & $9^{1}$ \\
\hline \multirow{5}{*}{83883} & $\mathrm{~L}$ & $5124.86^{5}$ & $5126.86^{5}$ & $5126.87^{5}$ & $1.68^{5}$ & $4.87^{5}$ & $0.71^{5}$ & $35^{5}$ \\
\hline & WL & $5101.58^{3}$ & $5105.58^{3}$ & $5105.61^{3}$ & $0.68^{2}$ & $0.37^{3}$ & $0.04^{1}$ & $17^{3}$ \\
\hline & PL & $5100.08^{2}$ & $5104.08^{2}$ & $5104.10^{2}$ & $0.59^{1}$ & $0.34^{1}$ & $0.05^{2}$ & $11^{1}$ \\
\hline & $\mathrm{TL}$ & $5111.68^{4}$ & $5115.68^{4}$ & $5115.70^{4}$ & $1.28^{4}$ & $2.62^{4}$ & $0.35^{4}$ & $28^{4}$ \\
\hline & TPL & $5097.86^{1}$ & $5103.86^{1}$ & $5103.92^{1}$ & $0.75^{3}$ & $0.35^{2}$ & $0.05^{3}$ & $14^{2}$ \\
\hline \multirow{5}{*}{83887} & $\mathrm{~L}$ & $5529.37^{5}$ & $5531.37^{5}$ & $5531.38^{5}$ & $2.31^{5}$ & $6.37^{5}$ & $1.21^{5}$ & $35^{5}$ \\
\hline & WL & $5520.61^{4}$ & $5524.61^{4}$ & $5524.64^{4}$ & $1.64^{3}$ & $3.11^{3}$ & $0.54^{3}$ & $25^{4}$ \\
\hline & PL & $5514.79^{2}$ & $5518.79^{2}$ & $5518.82^{2}$ & $1.36^{2}$ & $2.13^{2}$ & $0.33^{2}$ & $13^{2}$ \\
\hline & $\mathrm{TL}$ & $5516.47^{3}$ & $5520.47^{3}$ & $5520.50^{3}$ & $1.90^{4}$ & $3.91^{4}$ & $0.73^{4}$ & $23^{3}$ \\
\hline & TPL & $5510.67^{1}$ & $5516.67^{1}$ & $5516.72^{1}$ & $1.28^{1}$ & $1.83^{1}$ & $0.28^{1}$ & $9^{1}$ \\
\hline \multirow{5}{*}{83891} & 5 & $5345.73^{5}$ & $5347.73^{5}$ & $5347.74^{5}$ & $1.87^{5}$ & $5.55^{5}$ & $0.97^{5}$ & $35^{5}$ \\
\hline & WL & $5328.22^{3}$ & $5332.22^{3}$ & $5332.25^{3}$ & $1.11^{3}$ & $1.39^{3}$ & $0.23^{3}$ & $20^{3}$ \\
\hline & PL & $5324.89^{2}$ & $5328.89^{2}$ & $5328.92^{2}$ & $0.95^{2}$ & $0.97^{2}$ & $0.15^{2}$ & $13^{2}$ \\
\hline & $\mathrm{TL}$ & $5332.44^{4}$ & $5336.44^{4}$ & $5336.46^{4}$ & $1.45^{4}$ & $3.14^{4}$ & $0.55^{4}$ & $28^{4}$ \\
\hline & TPL & $5322.09^{1}$ & $5328.09^{1}$ & $5328.14^{1}$ & $0.92^{1}$ & $0.83^{1}$ & $0.13^{1}$ & $9^{1}$ \\
\hline \multirow{5}{*}{83920} & 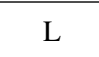 & $5170.00^{5}$ & $5172.00^{5}$ & $5172.01^{5}$ & $2.01^{5}$ & $5.97^{5}$ & $1.05^{5}$ & $35^{5}$ \\
\hline & WL & $5154.14^{3}$ & $5158.14^{3}$ & $5158.17^{3}$ & $1.12^{3}$ & $1.64^{3}$ & $0.27^{3}$ & $21^{3}$ \\
\hline & PL & $5148.31^{2}$ & $5152.31^{2}$ & $5152.34^{2}$ & $0.87^{2}$ & $1.01^{2}$ & $0.15^{2}$ & $13^{2}$ \\
\hline & $\mathrm{TL}$ & $5156.30^{4}$ & $5160.30^{4}$ & $5160.32^{4}$ & $1.62^{4}$ & $3.51^{4}$ & $0.62^{4}$ & $28^{4}$ \\
\hline & TPL & $5144.55^{1}$ & $5150.55^{1}$ & $5150.60^{1}$ & $0.79^{1}$ & $0.85^{1}$ & $0.12^{1}$ & $8^{1}$ \\
\hline \multirow{5}{*}{83923} & 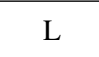 & $3980.20^{5}$ & $3982.20^{5}$ & $3982.21^{5}$ & $1.23^{5}$ & $1.15^{5}$ & $0.20^{5}$ & $31^{5}$ \\
\hline & $\mathrm{WL}$ & $3977.86^{4}$ & $3981.86^{3}$ & $3981.89^{3}$ & $0.86^{4}$ & $0.54^{3}$ & $0.09^{3}$ & $24^{4}$ \\
\hline & PL & $3977.42^{3}$ & $3981.42^{2}$ & $3981.46^{2}$ & $0.81^{2}$ & $0.52^{2}$ & $0.09^{2}$ & $16^{1}$ \\
\hline & $\mathrm{TL}$ & $3977.10^{2}$ & $3981.10^{1}$ & $3981.13^{1}$ & $0.84^{3}$ & $0.56^{4}$ & $0.10^{4}$ & $17^{2}$ \\
\hline & TPL & $3976.00^{1}$ & $3982.00^{4}$ & $3982.07^{4}$ & $0.68^{1}$ & $0.42^{1}$ & $0.07^{1}$ & $17^{2}$ \\
\hline
\end{tabular}



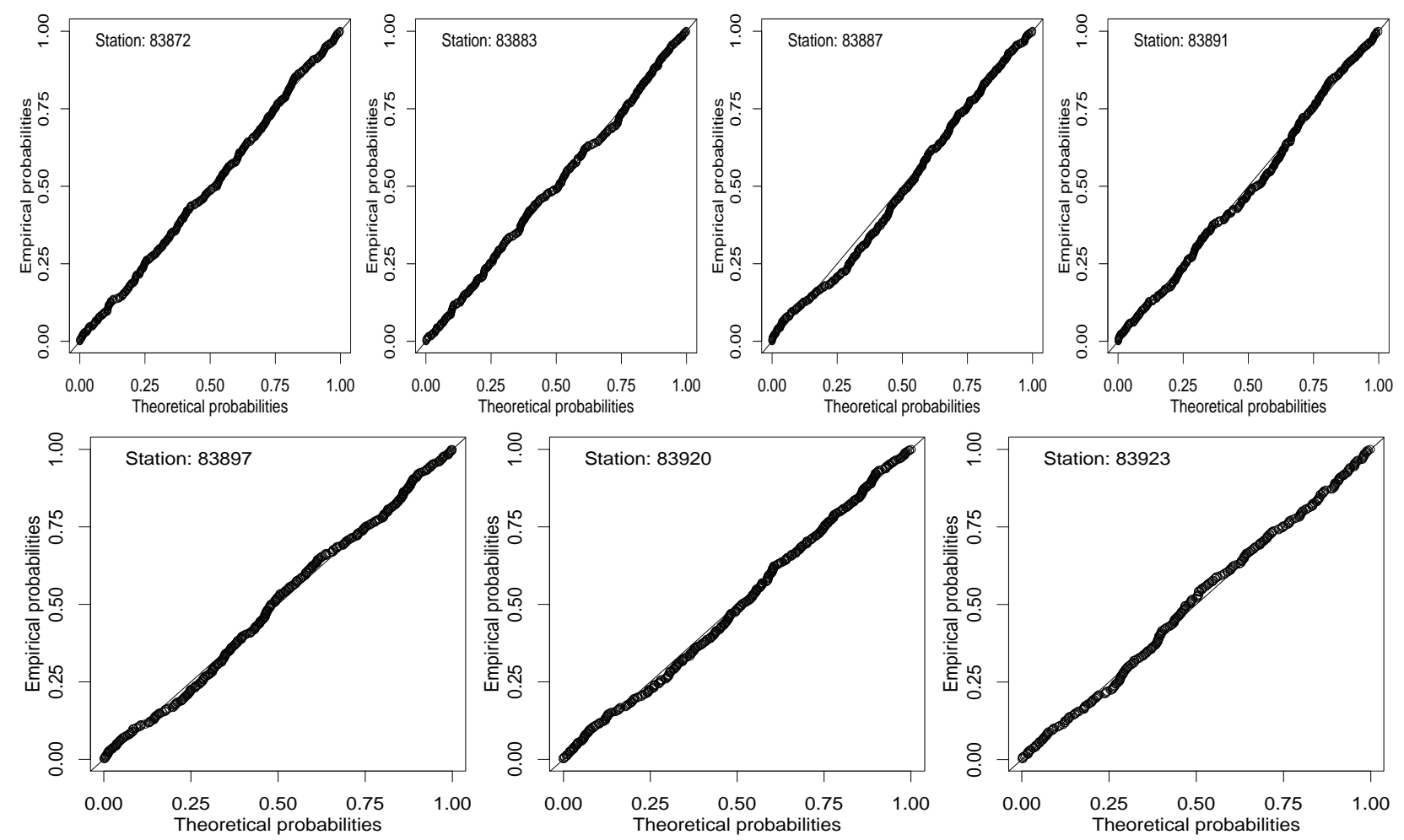

Figure 3. PPlot of the adjusted TPL model.

Table 5. Maximum likelihood and standard errors estimates for $\theta, \alpha$ and $\lambda$.

\begin{tabular}{cccc}
\hline Station & $\theta$ & $\alpha$ & $\lambda$ \\
\hline \multirow{2}{*}{83872} & 0.0224 & 0.9502 & -0.6632 \\
& $(0.0076)$ & $(0.0588)$ & $(0.1556)$ \\
\hline \multirow{2}{*}{83883} & 0.0183 & 0.9718 & -0.7973 \\
& $(0.0065)$ & $(0.0615)$ & $(0.1399)$ \\
\hline \multirow{2}{*}{83887} & 0.0047 & 1.0979 & 0.9786 \\
& $(0.0010)$ & $(0.0433)$ & $(0.0447)$ \\
\hline \multirow{2}{*}{83891} & 0.0049 & 1.1314 & 0.9647 \\
& $(0.0010)$ & $(0.0429)$ & $(0.0495)$ \\
\hline \multirow{2}{*}{83920} & 0.0043 & 1.1436 & 0.9621 \\
& $(0.0009)$ & $(0.0438)$ & $(0.0474)$ \\
\hline \multirow{2}{*}{83923} & 0.0275 & 0.9157 & -0.6510 \\
& $(0.0127)$ & $(0.0789)$ & $(0.2341)$ \\
\hline
\end{tabular}

\section{Conclusion}

In this paper we have introduced a new generalization of the power Lindley distribution, the transmuted power Lindley model. The proposed distribution was constructed by using a quadratic rank transmutation map and taking the power Lindley, which was formulated by using a power transformation $X=T^{1 / \alpha}$, as the baseline distribution. Some mathematical properties along with order statistics and estimation issues are addressed.

A real data was considered in order to illustrate the usefulness and effectiveness of the new model. In addition to the transmuted model, four different models were fitted and seven different statistics of fit were used as selection criteria: $-2 \times \log$-likelihood, AIC, AICC, BIC, KS, AD and CvM. 
Furthermore, in this paper we showed that the TPL model, despite having a small number of parameters, still interpretable (the key parameters of the power Lindley distribution is kept) and flexible, i.e, the TPL model can be used in several areas of application.

\section{Acknowledgments}

The authors are grafeful the Editorial Boarding as well as to the referees for their comments and suggestions. The research is partially funded by the Brazilian organizations CNPq and FAPESP.

\section{References}

Aryal, G. R., \& Tsokos, C. P. (2009). On the transmuted extreme value distribution with applications. Nonlinear Analysis, 71,1401-1407.

Aryal, G. R., \& Tsokos, C. P. (2011). Transmuted Weibull distribution: a generalization of the Weibull probability distribution. European Journal of Pure and Applied Mathematics, 4(2), 89-102.

Ghitany, M. E., Atieh, B., \& Nadarajah, S. (2008). Lindley distribution and its applications. Mathematics and Computers in Simulation, 78(4), 493-506.

Ghitany, M. E., Al-Mutairi, D. K., Balakrishnan, N., \& Al-Enezi, L. J. (2013). Power Lindley distribution and associated inference. Computational Statistics and Data Analysis, 20-33.

Granzotto, D. C. T., \& Louzada, F. (2014). The Transmuted Log-Logistic Distribution: Modeling, Iference and an application to a Polled Tabapua Race Time up to First Calving Data. Communications in Statistics - Theory and Methods (Accepted).

Lindley, D. V. (1958). Fiducial distributions and Bayes theorem. Journal of the Royal Statistical Society, 20(1), 102-107.

Louzada, F., \& Granzotto, D. C. T. (2015). The transmuted log-logistic regression model: a new model for time up to first calving of cows. Statistical Papers.

Merovci, F., \& Elbatal, I. (2014). Transmuted Lindley-Geometric Distribution and its Applications. Journal of Statistics Applications and Probability, 3(1), 77-91.

Nadarajah, S., Bakouch, H., \& Tahmasbi, R. (2011). A generalized Lindley distribution. Sankhya B, 73, 331-359.

SAS. (2011). SAS/ETSR® Userąŕs Guide, Version 9.33. Cary, NC: SAS Institute Inc.

Shaw, W. T., \& Buckley, I. R. C. (2007). The alchemy of probability distributions: beyond Gram-Charlier expansions, and a skew-kurtotic-normal distribution from a rank transmutation map. UCL Discovery Repository, pages 1-16.

Warahena-Liyanage, G., \& Pararai, M. (2014). A generalized power Lindley distribution with applications. Asian Journal of Mathematics ans Applications, 23.

Zakerzadeh, H., \& Dolati, A. (2009). Generalized Lindley Distribution. Journal of Mathematical Extension, 3(2), 13-25.

\section{Copyrights}

Copyright for this article is retained by the author(s), with first publication rights granted to the journal.

This is an open-access article distributed under the terms and conditions of the Creative Commons Attribution license (http://creativecommons.org/licenses/by/4.0/). 